\title{
Control of food intake during continuous injection of glucose into the upper duodenum and the upper ileum of rats
}

\author{
Z. GLICK and M. MODAN \\ Department of Nutrition, Hebrew University-Hadassah Medical School, Jerusalem, Israel \\ and \\ Department of Clinical Epidemeology, Chaim Sheba Medical Center, Tel-Hashomer, Israel
}

\begin{abstract}
Duodenal factors have been implicated to participate in the elicitation of satiety during a single meal. This report evaluates the possible involvement of the upper small intestines in the day-to-day control of food intake. Glucose solutions, contributing about a third of the daily caloric intake of rats, were continuously injected into the gastrointestinal tract through a permanent catheter. The catheter was inserted into each animal at one of two locations: the upper duodenum and the upper ileum. There were five animals in each catheter group. Voluntary food intake was measured before and during glucose injection. Control injections of oil equicaloric to glucose and $\mathrm{NaCl}$ equiosmotic to glucose were also performed. All glucose injections resulted in a reduction in voluntary food intake which began as early as the first day of injection and compensated for most of the caloric content of the injected glucose. The reduction was similar for the two catheter groups. Equicaloric injections of oil produced an effect similar to glucose, while equiosmotic $\mathrm{NaCl}$ injections did not. It is concluded that if there is an intestinal metering system which participates in day-to-day control of food intake, it is not exclusively located in the upper small intestines.
\end{abstract}

Satiety usually takes place before any significant absorption of nutrients occurs, suggesting that the gastrointestinal tract may play a role in inducing satiety, thus in controlling food intake. Evidence of adequate regulation of food intake in the absence of oropharingeal sensations (Epstein \& Teitelbaum, 1962) and after gastrectomy (McDonald, Ingelfinger, \& Belding, 1947) suggests that intestinal factors may be involved; continued regulation following vagotomy (Snowdon \& Epstein, 1970) or after complete gastrointestinal denervation (Grossman, Cummins, \& Ivy, 1947) points to a humoral mechanism. The participation of humoral factors in induction of satiety has, indeed, been reported in recent years (Davis, Campbell, Gallagher, \& Zurakov, 1971; Seoane, Baile, \& Martin, 1972).

Two approaches have been applied in the study of the role of intestinal factors in the control of food intake. In one, intestinal substances have been injected into hungry animals. Thus, intraperitoneal injections of cholecystokinin were found to reduce food intake of rats to varying extents under different experimental conditions (Glick, Thomas, \& Mayer, 1971; Smith, Gibbs, \& Young, 1974); however, its role in the normal control of food intake needs further exploration since systemic injections

This work was supported by a grant from the Israel Ministry of Health. We are grateful for the able and devoted technical assistance of Mrs. Ora Amado and Miss Ruth Levy. of physiological doses of this hormone were without effect (Glick et al., 1971). The other approach consists of injecting various substances directly into GI tracts of hungry animals. In these experiments, injection of glucose into the stomach or the duodenum prior to a meal of free-feeding animals, as well as those feeding under various schedules, reduced the size of the ensuing meal (Campbell \& Davis, 1974a, b; Ehman, Albert, \& Jamieson, 1971; Novin, Sanderson, \& Vanderweele, 1974; Yin \& Tsai, 1973). Injection of solutions of $\mathrm{NaCl}$, equi-osmotic to glucose (Yin \& Tsai, 1973; Ehman et al., 1971) or urea (Campbell \& Davis, 1974a, b) as well as injection of nonnutritive bulk (Ehman et al., 1971) also suppressed food intake.

Yin and Tsai (1973) and, more recently, Snowdon (1975) showed that intraduodenal injections of glucose during feeding, at concentrations of about $1 \mathrm{M}$, were more effective than intragastric injections in suppressing meal size. Furthermore, Snowdon (1975) showed that hypertonic nonnutritive solutions reduced meal size and increased frequency of feeding when injected intraduodenally but not intragastrically. These experiments suggest the possible existence of an intestinal satiety system which regulates the size of individual meals.

In addition, it has been shown that by continuous injections of a liquid diet for several days into the stomach (Epstein \& Teitelbaum, 1962), or a glucose solution into the duodenum (Snowdon, 1975), a compensatory adjustment in food intake occurs, but 
that during several days of injection of glucose solutions systemically, no such adjustment takes place (Adair, Miller, \& Booth, 1968; Glick et al., 1971). These findings indicate that intestinal factors may be involved in the day-to-day control of food intake, in addition to their proposed role in the control of meal size.

The experiment presented here was designed to evaluate whether the upper part of the small intestine is essential in a physiologic system, operative on a day-to-day basis, which is capable of metering the caloric contribution of nutrients reaching it, leading to maintenance of energy balance. For this purpose, glucose solutions, oil equicaloric to the glucose, and $\mathrm{NaCl}$ solutions equisomotic to the glucose were continuously injected for several days into two locations along the gastro-intestinal tract, i.e., upper duodenum and upper ileum, and adjustments in voluntary food intakes were measured. Obviously, if the GI tract proximal to the site of catheter insertion is the only intestinal component involved in a metering system, compensatory adjustment in food intake will be jeopardized, leading to an increased total caloric intake.

\section{METHOD}

\section{Animals and Facilities}

The experimental animals were female rats of the Charles River CD strain, kept at constant lighting after reaching about $140 \mathrm{~g}$ and weighing 190-230 $\mathrm{g}$ at the start of the experiment.

Throughout the experiment, the rats were caged individually and pressed a bar in order to obtain food pellets $(4.0 \times 3.3 \mathrm{~mm}$ 45-mg P. J. Noyes) released from a pellet dispenser. They ate ad lib and were in a temperature-controlled animal room kept at $24^{\circ} \pm 2^{\circ} \mathrm{C}$.

\section{Surgery}

For the purpose of continuous injection of liquids into unrestrained rats, a modification of the system reported by Epstein

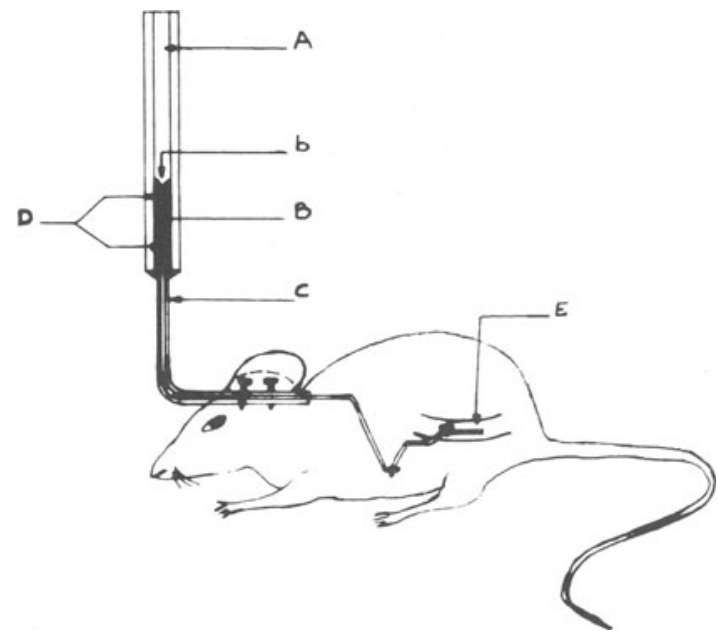

Figure 1: A schematic presentation of the preassembled catheter system as it was implanted in the rat. $A$, Tygon tubing; B, PE50 catheter; b, cone shape ending of catheter; $C$, metal guide; D, epoxy glue; E, gastrointestinal tract.
(1960) was used. The animals were permanently catheterized either in the duodenum $(1-2 \mathrm{~cm}$ from the pyloric sphincter) or at a distance of $40-42 \mathrm{~cm}$ distal to the pyloric sphincter. The catheters were introduced into the GI tract through the peritoneal cavity. A schematic presentation of the preassembled catheter and attachments unit as implanted in the rat is shown in Figure 1. The PE 50 catheter was inserted into the L-shaped guide and glued to the two ends of the guide, in order to prevent leakage of fluid around the catheter. At one end of the guide, a Tygon tube was attached, and the end of the catheter was inside this tube. At the other end of the guide, the catheter protruded freely. Each rat was fasted 1 day prior to the catheterization procedure and was anesthetized with Nembutal (veterinary, Abbot Lab.) before the operation. After exposing the posterior portion of the skull by a midline incision, the intestinal tract was exposed by another 6-cm midline incision beginning immediately below the xyphoid. The free end of the catheter was led under the skin via a stainless steel needle guide through the incision in the skull to the peritoneal cavity (Figure 1), and cut to fit the desired site of implantation. A distance of $40-42 \mathrm{~cm}$ on the small intestines was determined by bending a premeasured thread of such length along the intestinal folds, beginning from the pylorus.

Prior to implantation of the catheter into the intestine, the following procedures were performed: (1) In order to prevent the catheter from sliding out of the intestine, a square knot was made with a cellulose thread $1 \mathrm{~cm}$ from the free end of the catheter; the knot was tight, but allowed free passage of injected liquids. (2) In order to prevent distortion of the natural folds of the intestines when the implanted catheter was sutured to the peritoneal muscles, a $90^{\circ}$ angle was formed on the catheter at a distance of $2 \mathrm{~cm}$ from its free end by inserting it in boiling water.

The free end of the catheter was then led into the GI tract in the distal direction through a $2-\mathrm{mm}$ longitudinal incision in the gastrointestinal wall until the square knot was just within the lumen. The incision was then sutured twice with a fine needle (5/0 F595 Mersutures eyeless needle suture, Ethicon, Ltd.). The position of the catheter was secured by a suture to the peritoneal muscle, and then the abdomen was closed.

The metal guide was secured to the skull by a cranioplast of dental cement supported by three screws, as shown in Figure 1. A swivel joint system to allow free movement of the rats, as developed by Thomas and Mayer (1968), was attached to the Tygon tube approximately $7 \mathrm{~cm}$ above the skull.

The rats were injected with penicillin immediately following the operation and daily for the next 6 days.

The animals were fasted $24 \mathrm{~h}$ after the operation. They regained preoperation weights within 7-8 days, and then fed ad lib. Experimental data were collected only after the rats showed constant rates of weight gain.

\section{Design and Analysis}

Voluntary food intake was measured in each animal during a pretreatment control period in which nothing was injected through the catheter. This period started about 14 days after the operation and lasted 3-8 days. Subsequently, each animal was continuously injected for at least 3 days, with the aid of a syringe pump, with one of the following liquids:

(1) A solution of $30 \%$ glucose, at a rate of $22 \mathrm{ml} / 24 \mathrm{~h}$, contributing $24.4 \mathrm{Cal}$ per day, which was about a third of the rats' normal daily voluntary caloric intake. This treatment was given to 10 rats, 5 with a duodenal and 5 with an ileal catheter.

(2) Soy-bean oil at a rate of $3.1 \mathrm{ml} / 24 \mathrm{~h}$, contributing the same amount of energy as the glucose. This was injected into 7 animals, 4 with a duodenal and 3 with an ileal catheter.

(3) A solution of $5.4 \% \mathrm{NaCl}$, isoosmotic to the glucose and injected at the same rate. This solution was administered to 6 rats, 3 with a duodenal and 3 with an ileal catheter.

The rats stayed attached to the swivel joint system and were weighed daily throughout the experiment. After collection of the data, the rats were sacrificed to validate that the catheter was still properly placed. 


\begin{abstract}
The mean voluntary daily caloric intake (MVDCI), as recorded by the barpress, was calculated for each period of treatment. Results were expressed as the percent change of the individual MVDCI during administration of the various solutions from the MVDCI of the control (preinjection) period. Results were evaluated statistically by a two-way analysis of variance.

In order to test whether there was a backflow of infusate from the upper ileum to the duodenum, four additional rats were catheterized in the upper ileum and injected as described earlier with a $30 \%$ glucose solution into which an Evans blue dye was dissolved at a final concentration of $0.12 \%$. Two rats were sacrificed $10 \mathrm{~h}$ and two $20 \mathrm{~h}$ after the beginning of injection. Their intestines were divided into 10 to $15-\mathrm{cm}$-long segments, and their contents washed out and diluted to a final volume of $25 \mathrm{ml}$ with distilled water.
\end{abstract}

\section{RESULTS}

Individual and mean percent change in the mean voluntary daily caloric intake (MVDCI) compared to the pretreatment level are shown in Figure 2 for the three injection groups $(30 \%$ glucose, $5.4 \% \mathrm{NaCl}$, and oil) and for the two sites of injection.

All 17 animals that received injections of energycontributing solutions, i.e., glucose or oil, showed without exception a considerable decrease in voluntary food intake immediately after the injection was started, while in the animals that received $5.4 \% \mathrm{NaCl}$ no such reduction occurred.

The decrease in the mean MVDCI of the animals injected with glucose or oil compared to the preinjection period was highly significant $[p(t)<.001]$ and similar with both treatments $[p(t)>.50]$. The MVDCI during administration of the $\mathrm{NaCl}$ solution did not differ significantly from control values $[p(t)>.50]$. For animals in which the catheter was inserted in the duodenum or ileum, there was no difference between the mean MVDCI during injection of glucose or oil $[p(F)>.40]$.

The mean decrease in MVDCI following nutrient injection was $34.7 \mathrm{Cal}$ with a SD of $11.3 \mathrm{Cal}$. The reduction of MVDCI thus exceeded the caloric contribution of the nutrients by $10 \mathrm{Cal}$ on the average. Nevertheless, during the injection, weight changes of each animal were similar to those during the control period.

In the control dye-injection experiment, no blue dye was observed in the upper $30 \mathrm{~cm}$ of the small intestines, and only a small quantity of it was seen in the 10 -cm-long segment immediately proximal to the site of the catheter insertion, mostly concentrated near this site. The rest of the intestines distal to the site of injection, including ileum, cecum, and large bowel, were heavily pigmented with the blue dye. These results are clearly demonstrated in Figure 3. A colored picture would represent this finding more precisely, as a light shade of blue was visible in the third tube from the left. Thus it was concluded that a back-flow movement of the injected solutions from the ileum to the duodenum did not take place in any significant quantity.

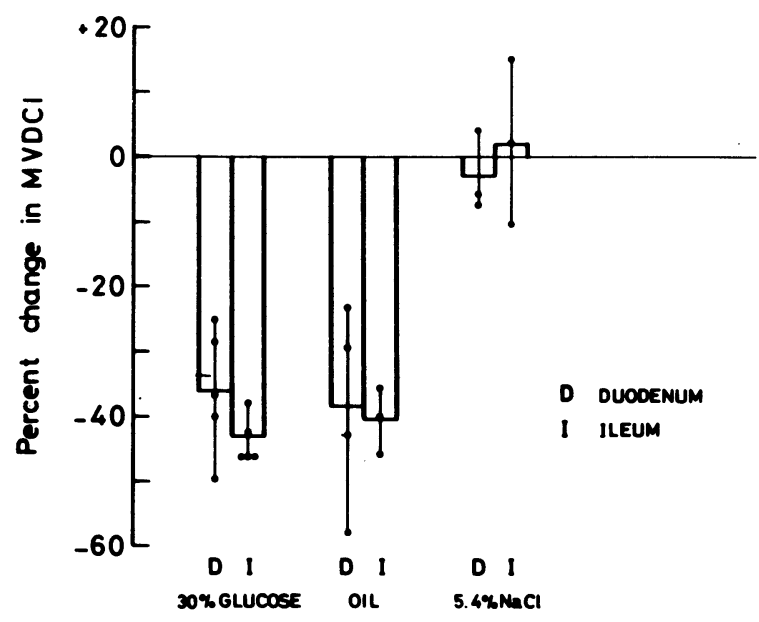

Figure 2. Individual and mean percent change in mean daily voluntary caloric intake compared to preinjection level in the three injection groups, i.e., $30 \%$ glucose, oil, and $5.4 \% \mathrm{NaCl}$ by catheter site.

\section{DISCUSSION}

The results of this study indicate that continuous injection of a $30 \%$ glucose solution or an equicaloric amount of oil, which is nonosmotic, into the duodenum or ileum causes a decrease in the total daily voluntary food intake which takes place as early as the first day of injection. This reduction is of the same order of magnitude for both nutrient sources, namely glucose and oil, and both catheter sites. Injection of $\mathrm{NaCl}$ equiosmotic to the glucose solution has no such effect.

Our results indicate that the reduction in mean daily voluntary food intake in the present experiment, which is concerned with the day-to-day control of food intake, is due to the caloric content of the injected nutrients and not due to an osmotic or bulk effect which elicits a satiety response during a single meal (Campbell \& Davies, 1974a, b; Ehman et al., 1971; Yin \& Tsai, 1973). These findings are compatible with those of Snowdon (1975) showing that daily injections of energy contributing nutrients into the duodenum of rats result in a compensatory decrease of voluntary food intake. We found, in addition, that such a compensatory decrease occurs even when the duodenum is bypassed. Thus it can be concluded that intestinal cues which may be associated with a control mechanism for daily food intake do not originate exclusively in the upper small intestines. Our findings suggest that cholecystokinin is dispensible in the day-to-day control of food intake, the upper intestine being the main site of its secretion.

It can be concluded that if, indeed, intestinal factors participate in the day-to-day control of food intake, any segment along the small intestine can play a role. If, on the other hand, intestinal factors do not participate in such a control mechanism, then the 


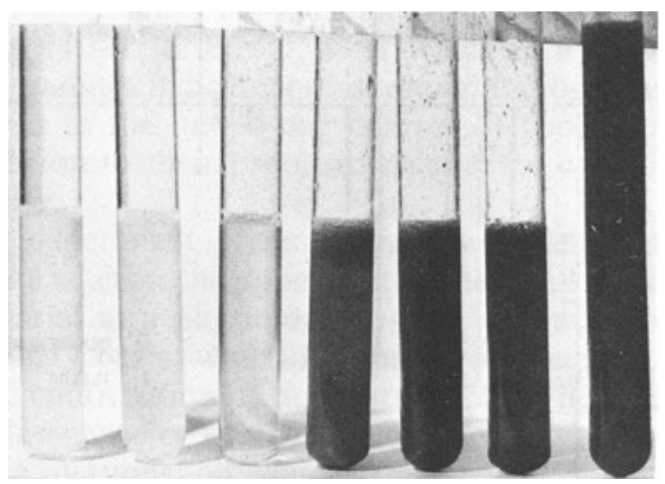

Figure 3. Evans blue content of intestinal segments following a 20-h continuous injection of the dye. Left to right is proximal to diastal sequence. The fourth tube from the left represents the segment immediately diastal to the site of catheter insertion. The last tube on the right contains cecum contents in twice the volume of water.

possibility of the involvement of hepatic receptors in the day-to-day control of food intake is raised. This is based on our present findings, as well as Snowdon's (1975), that compensatory adjustments in food intake occur during several days' injection of glucose into the small intestine, but not systemically into the right auricle (Adair et al., 1968) or aorta (Glick et al., 1971). Data on the effect of several days' injection of nutrients into the portal system are, to the best of our knowledge, not available at present, thus the possibility for such a role to the liver in the day-to-day control of food intake needs further experimental evidence. That liver receptors are important in controlling meal size has been proposed by Russek (1963) and has received further experimental support (Campbell \& Davis, 1974b; Russek, 1970; and others). In other studies, the involvement of liver receptors in controlling meal size could not be detected (Baile, Zinn, \& Mayer, 1971; Stephens \& Baldwin, 1974).

It is of interest that, during nutrient injections in the present study, the reduction in mean daily voluntary food intake in both catheter groups was more than the energy content of the injected nutrients. The reason for this is not entirely clear, since the animals did not lose weight during this period, but actually showed daily weight changes that were similar to those of the control period. The excess reduction in caloric intake may be a result of a decrease in activity associated with the attachment of the suspended injection system.

\section{REFERENCES}

Adair, E. R., Miller, N. E., \& Booth, D. A. Effects of continuous intravenous infusion of nutritive substances on con- sumatory behavior in rats. Communications in Behavioral Biology, 1968, 2, 25-37.

BAILE, C. A., ZinN, W., \& MAYER, J. Feeding behavior of monkeys: Glucose utilization rate and site of glucose entry. Physiology and Behavior, 1971, 6, 537-541.

Campbell, C. S., \& Davis, J. D. Peripheral control of food intake: Interaction between test diet and postingestive chemoreception. Physiology and Behavior, 1974, 12, 377-384. (a)

CAMPBEll, C. S., \& Davis, J. D. Licking rate of rats is reduced by intraduodenal and intraportal glucose infusion. Physiology and Behavior, 1974, 12, 357-365. (b)

Davis, J. D., Campbell, C. S., Gallagher, R. J., \& Zurakov, M. A. Disappearance of a humoral satiety factor during food deprivation. Journal of Comparative and Physiological Psychology, 1971, 75, 476-482.

Ehman, G. K., Albert, D. J., \& Jamieson, J. L. Injections into the duodenum and the induction of satiety in the rat. Canadian Journal of Psychology, 1971, 25, 147-166.

Epstein, A. N., \& Teitelbaum, P. Regulation of food intake in the absence of taste, smell and other oropharingeal sensations. Journal of Comparative and Physiological Psychology, 1962, 55, 753-759.

Epstern, A. N. Water intake without the act of drinking. Science, 1960, 131, 497-498.

Glick, Z., Thomas, D. W., \& Mayer, J. Absence of effect of injections of the intestinal hormones secretin and cholecystokinin-pancreozumin upon feeding behavior. Physiology and Behavior, 1971, 6, 5-8.

Grossman, M. L., Cummins, G. M., \& Ivy, A. C. The effect of insulin on food intake after vagotomy and sympathectomy. American Journal of Physiology, 1947, 149, 100-102.

MacDonald, R. M., Ingelfinger, F. J., \& Belding, H. W. Late effects of total gastrectomy in man. New England Journal of Medicine, 1947, 237, 887-896.

Novin, D., Sanderson, J. D., \& Vanderweele, D. A. The effect of isotonic glucose on eating as a function of feeding condition and infusion site. Physiology and Behavior, 1974, 13, 3-7.

RUSSEK, M. Participation of hepatic glucoreceptors in the control of intake of food. Nature, 1963, 197, 79-80.

RUSSEK, M. Demonstration of the influence of an hepatic glucosensitive mechanism on food intake. Physiology and Behavior, 1970, 5, 1207-1209.

Seoane, J. R., Baile, C. A., \& Martin, F. Humoral factors modifying feeding behavior of sheep. Physiology and Behavior, 1972, 8, 993-995.

Smith, G. P., Gibbs, J., \& Young, R. C. Cholecystokinin and intestinal satiety in the rat. Federation Proceedings, 1974, 33, 1146-1149.

SNowdon, C. T. Production of satiety with small intraduodenal infusions in the rat. Journal of Comparative and Physiological Psychology, 1975, 88, 231-238.

Snowdon, C. T., \& Epstein, A. N. Oral and intragastric feeding in vagotomized rats. Journal of Comparative and Physiological Psychology, 1970, 71, 59-67.

Stephens, D. B., \& Baldwin, B. A. The lack of effect of intrajugular or intraportal injections of glucose or amino-acids on food intake in pigs. Physiology and Behavior, 1974, 12, 923-929.

Thomas, D. W., \& MAYER, J. A simple and inexpensive swivel joint for the infusion of unrestrained animals. Physiology and Behavior, 1968, 3, 499-500.

YIN, T. H., \& TsAI, C. T. Effects of glucose on feeding in relation to routes of entry in rats. Journal of Comparative and Physiological Psychology, 1973, 85, 258-264.
(Received for publication June 15, 1976; revision accepted September 28, 1976.) 